\title{
Three Cases of Major Depression: Different Pharmacological Antidepressant Options
}

\section{Felix-Martin Werner ${ }^{1,2 *}$ and Rafael Covenas ${ }^{2}$}

${ }^{1}$ Higher Vocational School of Elderly Care and Occupational Therapy, Euro Academy Pößneck, Pößneck, Thuringia, 07381, Germany ${ }^{2}$ Laboratory of Neuroanatomy of the Peptidergic Systems (Lab. 14), Institute of Neurosciences of Castilla y León (INCYL), University of Salamanca, Castilla-León, 37007-Salamanca, Spain

\begin{abstract}
Three cases with different forms of major depression and different pharmacological antidepressant treatments are presented. In the first case report, citalopram, a selective serotonin reuptake inhibitor, improved transient depressive symptoms in a professionally busy man who recovers from the depressive symptoms and solves the social problems in his team. In the second case report showing a severe form of major depression and a suicide attempt, the tricyclic antidepressant drug doxepin failed to improve depressive symptoms. The selective serotonin and noradrenaline reuptake inhibitor venlafaxine partly improved depressive symptoms. Lithium carbonate, a prophylactic drug, which shows antidepressant and antimanic effects, treated depressive symptoms successfully. The cardiac and renal adverse effects were controlled. In the third case report, citalopram was used to treat the chronic form of major depression; however the adverse effects such as sedating and drive-reducing effects and the weight gain were disturbing for the patient. The administration of the selective dopamine and noradrenaline reuptake inhibitor bupropion ameliorated depressive symptoms and sleepiness, however caused hypertension and insomnia. Due to this changed medication, the patient succeeded in coping with a stressful life event, an anti-cancer therapy.
\end{abstract}

Keywords: Antidepressant drug; Bupropion; Clomipramine; Doxepin; Lithium; Major depression; Selective dopamine and noradrenaline reuptake inhibitor; Selective serotonin reuptake inhibitor; Tricyclic antidepressant drug; Venlafaxine

\section{Introduction}

Major depression is a frequent psychiatric disease with a prevalence of $7 \%$ and is associated with a prolonged sadness, loss of energy, interest and pleasure, reduced drive, alterations of the circadian rhythm with an early wakening and an improved mood in the evening. In this disease, hypoactivity of monoamines, i.e. serotonin, noradrenaline and dopamine occurs in the brainstem and hippocampus [1]. Three case reports of major depression are presented showing different degrees of depressive symptoms and pharmacotherapy [2]. Thus, a transient moderate form of major depression was treated by the selective serotonin reuptake inhibitor citalopram [3]. A severe form of major depression was treated by the selective serotonin and noradrenaline reuptake inhibitor venlafaxine and lithium [4,5]. A severe chronic form of major depression was treated by the selective dopamine and noradrenaline reuptake inhibitor bupropion [6].

\section{Case Report 1}

A 44-year old bank employer, who is married and has two daughters, complains about stress at work and social problems in the team, he shows a reduced activity and alterations of the circadian rhythm with an early wakening. After a busy working day, he expresses thoughts of suicide. The physician diagnoses a moderate form of major depression and prescribes the selective serotonin reuptake inhibitor citalopram with a dosage of $20 \mathrm{mg}$ once daily and gives a health insurance certificate for three weeks. The complains with the early wakening are improved and the patient passes the free time doing physical exercises. Adverse effects of citalopram were changes in blood pressure, constipation and disturbances of the sight. The E.C.G. is registered, and it showed no abnormalities. After three weeks, the patient starts working again, in the working team the problems of social interactions are talked about and are solved in the next weeks. During the next weeks, the patient shows no recurrence of depressive symptoms.

\section{Case Report 2}

A 61-year old caretaker in a residential home for foreigners, who suffers from a diabetes mellitus type 2 (he needs two injections of mixed insulin per day), shows a reduced activity and a prolonged sadness, once he was divorced. He has more and more difficulty to perform the work. He is admitted in the emergency department of the hospital and later he tries suicide with an overdose injection of insulin. In the hospital, he is transferred to a psychiatric ward and an antidepressant pharmacotherapy is started. After the administration of the tricyclic antidepressant drug doxepine (50 $\mathrm{mg}$ per day) the depressive symptoms did not improve, and then the selective serotonin and noradrenaline reuptake inhibitor venlafaxine ( $75 \mathrm{mg}$ per day) was prescribed. This antidepressant drug does not improve completely the depressive symptoms. Finally, the prophylactic drug lithium carbonate (675 mg per day) is administered and depressive symptoms are greatly ameliorated. The E.C.G. is recorded regularly and the kidney parameters are controlled. The patient leaves the hospital showing more and more social and physical activities. The patient is retired after leaving the hospital.

*Corresponding author: Werner FM, Medical Doctor, $\mathrm{Dr}$. Med, Research Field: Neural Networks in Neurological and Psychiatric Diseases, University of Salamanca, Instituto de Neurociencias de Castilla y León (INCYL), Laboratorio de Neuroanatomía de los Sistemas Peptidérgicos (Lab. 14), c/ Pintor Fernando Gallego, 1, 37007-Salamanca, Spain, Tel: +34/923/294400; extn. 1856; Fax: +34/923/29 45 49; E-mail: felixm-werner@versanet.de

Received June 01, 2017; Accepted June 20, 2017; Published June 28, 2017

Citation: Werner FM, Covenas R (2017) Three Cases of Major Depression Different Pharmacological Antidepressant Options. J Cytol Histol 8: 457. doi: 10.4172/2157-7099.1000457

Copyright: (c) 2017 Werner FM, et al. This is an open-access article distributed under the terms of the Creative Commons Attribution License, which permits unrestricted use, distribution, and reproduction in any medium, provided the original author and source are credited. 


\section{Case Report 3}

A 63-year old nurse working in a children's hospital cares her father who is suffering from Alzheimer's disease. After her father's death, she develops more and more symptoms such a prolonged sadness and alterations of the circadian rhythm and has difficulties to perform her work. Once she is admitted in the hospital, the depressive symptoms are treated with an infusion of clomipramine at a dosage of $50 \mathrm{mg}$ per day, a drive increasing antidepressant drug. After the depressive symptoms are improved, she is treated with the selective serotonin reuptake inhibitor citalopram ( $40 \mathrm{mg}$ per day). With this medication, she complains about a raised sleepiness and a decreased drive. Besides, she shows weight gain, constipation and an increased intraocular pressure. The E.C.G. is controlled regularly. After one year, a trial to perform the work again fails so that she decides to retire. She shows social and physical activities again. Considering these symptoms, the physician prescribes the selective dopamine and noradrenaline reuptake inhibitor bupropion (150 mg per day). This antidepressant medication is possible because the patient has no tendency to develop neither psychotic nor manic symptoms. With this new medication, the patient is more active; however she suffers of some nights of insomnia. Hypertension is treated with an oral adminstration of ramipril (5 $\mathrm{mg}$ per day). Some years later, the patient is diagnosed with a colon carcinoma of the colon ascendens with the classification T3N2M0 and is successfully treated with hemicolectomy and chemotherapy. Once the anti-cancer therapy is finished, the depressive symptoms are stable and the patient continues the antidepressant pharmacotherapy with bupropion.

\section{Antidepressant pharmacotherapy}

Antidepressant drugs are monoamine reuptake inhibitors prolonging the effect of the monoamines in the synaptic cleft. Through this mechanism of action, depressive symptoms are improved. Antidepressant drugs have different mechanisms of action, improve the depressive symptoms to a different extent and exert different adverse effects [7].

\section{Tricyclic antidepressant drugs}

Tricyclic antidepressant drugs are serotonin and noradrenaline reuptake inhibitors. In the case report 2 , the tricyclic antidepressant drug doxepin is administered. It has antidepressant and sedative effects, but in this case report it fails to improve depressive symptoms [8]. The adverse effects of doxepin are somnolence, changes in blood pressure, constipation, an increased intraocular pressure and changes in the E.C.G. In the case report 3, the tricyclic antidepressant drug clompramine is administered in order to treat the acute depression. Clompramine has a drive-activating effect and improves the acute depressive symptoms [9].

\section{Selective serotonin reuptake inhibitors (SSRI's)}

SSRI's such as citalopram, fluoxetine and paroxetine exert antidepressant effects and, to a small extent, sedating effects [10]. Adverse effects are decreased blood pressure, constipation, increased intraocular pressure, weight gain and decreased sexual activity [7]. In the case report 1 , the transient depressive symptoms were improved after administration of citalopram.

\section{Selective serotonin and noradrenaline reuptake inhibitors (sSNRI's)}

Venlafaxine is a sSNRI that exerts a good antidepressant effect. In the case report 2, it improves partly the severe depressive symptoms. The adverse effects of venlafaxine are changes in the E.C.G., hypo/ hyper-tension, increased intraocular pressure, weight gain or loss and insomnia [7].

\section{Selective dopamine and noradrenaline reuptake inhibitors (DNRI's)}

In case report 3, the DNRI bupropion exerted a good antidepressant effect and improved depressive symptoms so that the patient could get over another stressful event, i.e. an anti-cancer therapy. This antidepressant drug improves decreased positive effects, i.e. loss of energy, interest and pleasure. Bupropion may not be administered in patients with manic or psychotic symptoms, because it can induce dopamine hyperactivity and thus worsen manic or psychotic symptoms [6]. Adverse effects are changes in the E.C.G., hypertension, insomnia, increased intraocular pressure and weight loss [7]. The patient in the case report 3 showed an increased blood pressure (treated with ramipril) and partly insomnia [2].

\section{Lithium}

In affective symptoms, lithium has a prophylactic effect. It decreases the effect of excitatory neurotransmitters such as dopamine and glutamate and increases the inhibitory effect of gamma-aminobutyric acid (GABA) [5]. In the case report 2, lithium carbonate improved depressive symptoms very much. The adverse effects of lithium are cardio- and nephro-toxicities. That is why lithium levels should be monitored regularly.

\section{Discussion}

Currently, there are different antidepressant drugs available for the treatment of major depression. SSRI's (e.g., citalopram) have a good antidepressant effect and in the case report 1 they successfully treated the transient depressive symptoms. Adverse effects such as arrhythmias, hypotension, increased intraocular pressure and constipation should be controlled [7]. Doxepin, a tricyclic antidepressant drug, inhibits the reuptake of serotonin and noradrenaline and in the case report 2 it fails to improve severe depressive symptoms. The sSNRI venlafaxine partly improves severe depressive symptoms. Consequently, venlafaxine has a higher antidepressant effect than tricyclic antidepressant drugs. Venlafaxine has slightly different adverse effects in comparison to SSRIs; it can cause hypo- and hypertension, insomnia and weight loss and gain [4]. In the psychiatric ward, the physician decided to administer lithium carbonate, a prophylactic drug, which definitely improved depressive symptoms. The question why one third of patients with affective symptoms treated with lithium recover from these symptoms is not yet answered [5].

In case report 3 , the patient complains about sleepiness and a reduced drive while taking citalopram as an antidepressant pharmacotherapy. Since the patient does not show manic or psychotic symptoms, the physician decides to prescribe the selective DNRI bupropion. With the changed medication, the patient is more active, however shows days of insomnia which is treated by a hypnotic. The novel antidepressant drug enables the patient to cope with another stressful life event, i.e. an anticancer therapy. Insomnia, hypertension and weight loss are atypical adverse effects of this novel antidepressant drug [7].

\section{Conclusion}

For the treatment of different forms of major depression, different pharmacological groups of antidepressant drugs are avaible. Tricyclic 
Citation: Werner FM, Covenas R (2017) Three Cases of Major Depression: Different Pharmacological Antidepressant Options. J Cytol Histol 8: 457. doi: $10.4172 / 2157-7099.1000457$

antidepressant drugs, which inhibit the reuptake of the monoamines serotonin and noradrenaline, exert antidepressant, sedating and driveactivating effects. Tricyclic antidepressant drugs, such as clomipramine, which inhibits the reuptake of serotonin and noradrenaline, can be used to treat acute depressive symptoms. The tricyclic antidepressant drug, doxepin, does not improve acute depressive symptoms in severe forms of major depression. The SSRI citalopram can improve transient depressive symptoms in a moderate form. The professionally busy patient recovers totally from the depressive episode. However, for the treatment of a chronic severe form of major depression, its adverse effects such as sedating and the drive-reducing effects are disturbing for the patient. In one third of patients, lithium carbonate can ameliorate severe depressive symptoms. It inhibits the effect of neurotransmitters such as dopamine and glutamate and enhances the presynaptic inhibitory effect of GABA. Its antidepressant effect can be seen very clearly when the cardiac and renal adverse effects were controlled. A severe form of major depression with a suicide attempt could be resolved with this medication. Bupropion, a selective DNRI that inhibits the reuptake of dopamine and noradrenaline can be used to treat severe forms of major depression in patients who do not show psychotic nor manic symptoms and to treat the loss of energy, interest and pleasure.

\section{Conflicts of Interest}

Authors declared no conflict of interest.

\section{References}

1. Werner FM, Coveñas R (2013) Classical neurotransmitters and neuropeptides involved in major depression in a multi-neurotransmitter system: focus on antidepressant drugs. Curr Med Chem 20: 4853-4858.

2. Werner FM, Coveñas R (2014) Efficacy of novel antidepressant drugs in comparison to onventional antidepressant drugs. J Clin Case Rep 4: 11.

3. Navines R, Martín-Santos R, Gómez-Gil B, Martínez de Osaba MJ, Imaz ML, et al. (2007) Effects of citalopram treatment on hypothermic and hormonal responses to the $5-\mathrm{HT}(1 \mathrm{~A})$ receptor agonist buspirone in patients with major depressin and therapeutic response. Psychoendocrinology 32: 307-312.

4. Signorovitch J, Ramakrishnan K, Ben-Hamadi R, Yu AP, Wu EQ, et al. (2011) Remission of major depressive disorder without adverse events: a comparison of escitalopram versus serotonin norepinephrine reuptake inhibitors. Curr Med Res Opin 27: 1089-1096.

5. Malhi GS, Tanious M, Coulston CM, Berk (2013) M Potential mechanisms of action of lithium in bipolar disorder: current understanding. CNS Drugs 27: 135-153.

6. Sennfelt DA, Marques da Silva MA, Tavares AP (2011) Bupropion in the treatment of major depressive disorder in real-life practice. Clin Drug Investig 31: 19-24.

7. Werner FM, Coveñas R (2016) Additional Antidepressant Pharmacotherapies According to A Neural Network. Brain Disord Ther 5: 203.

8. Yeung WF, Chung KF, Yung KP, Ng TH. (2015) Doxepin for insomina: systematic review of randomized placebo-controlled trials. Sleep Med Rev 19: 75-83

9. Thomas SJ, Shin M, Mclnnis MG, Bostwick JR (2015) Combination therapy with monamine oxidase inhibitors and other antidepressant or stimulants: strategies for the management of treatment-resistant depression. Pharmacotherapy 35 : 433-449.

10. Marek GJ, Day M, Hudzik TJ (2015) The utility of impulse bias and altered decision-making as predictors of drug efficacy and target selection: Rethinking behavioral screening for antidepressant drugs. J Pharmacol Exp Ther 356(3): 534-548. 